\title{
Comparative performances of prognostic indexes for breast cancer patients presenting with brain metastases
}

\author{
Antoine-Laurent Braccini ${ }^{*}$, David Azria ${ }^{1}$, Simon Thezenas ${ }^{2}$, Gilles Romieu ${ }^{3}$, Jean-Marc Ferrero ${ }^{4}$ and William Jacot ${ }^{3}$
}

\begin{abstract}
Background: Several prognostic indexes (PI) have been developed in the brain metastases (BM) setting to help physicians tailor treatment options and stratify patients enrolled in clinical studies. The aim of our study was to compare the clinical relevance of the major PI for breast cancer BM.

Methods: Clinical and biological data of 250 breast cancer patients diagnosed with BM at two institutions between 1995 and 2010 were retrospectively reviewed. The prognostic value and accuracy of recursive partitioning analysis (RPA), graded prognostic assessment (GPA), basic score for BM (BS-BM), breast RPA, breast GPA, Le Scodan's Score and a clinico-biological score developed in a phase I study (P1PS) were assessed using Cox regression models. PI comparison was performed using Harrell's concordance index.

Results: After a median follow-up of 4.5 years, median overall survival (OS) from BM diagnosis was 8.9 months (Cl 95\%, 6.9-10.3 months). All PI were significantly associated with OS. Harrell's concordance indexes C favored BS-BM and RPA. In multivariate analysis, the RPA, Le Scodan's score and GPA were found to be the best independent predictors of OS. In multivariate analysis restricted to the 159 patients with known LDH and proteinemia, RPA 2 and 3, Le Scodan's Score 3 and P1PS 2/3 were associated with worse survival. RPA was the most accurate score to identify patients with long (superior to 12 months) and short (inferior to 3 months) life expectancy.
\end{abstract}

Conclusions: RPA seems to be the most useful score and performs better than new PI for breast cancer BM.

Keywords: Breast cancer, Brain metastases, Prognostic indexes, Biological subtype

\section{Background}

The Recursive Partitioning Analysis RPA [1] was the first prognostic score developed in the brain metastases (BM) setting. This classification was created in 1997 by the Radiation Therapy Oncology Group after analysis of the relative contributions of pretreatment variables to survival of patients with BM. Since this date, several scores and prognostic indexes (PI), such as the Graded Prognosis Assessment (GPA) [2], the Basic Score for BM (BS-BM) [3], the Phase 1 Prognostic Score (P1PS) [4], the Rotterdam score [5], the Score Index for Radiosurgery (SIR) [6] and the Rades's score [7] have been developed both to help physicians tailor treatment options depending on patient

\footnotetext{
* Correspondence: Antoine.Braccini@montpellier.unicancer.fr

${ }^{1}$ Department of Radiation Oncology, Val d'Aurelle Cancer Institute, 208 rue des apothicaires, Montpellier 34298, France

Full list of author information is available at the end of the article
}

prognosis, and to stratify patients enrolled in clinical studies. However, it has been demonstrated that the prognostic value of these scoring systems differs according to the primary tumor site [8], which raises the question of the usefulness of a breast-specific score.

Breast cancer is the second cause of BM, after lung cancer. Breast cancer is a heterogeneous disease with metastatic pattern and survival varying with the expression of biological markers such as the hormonal receptor (HR) status and human epidermal growth factor receptor-2 (HER2) overexpression. While the incidence of BM from breast cancer has increased over the past decade, especially for the subgroup of HER2-overexpressing tumors, several studies have shown that biological subtypes influence survival, even after BM diagnosis. In a series of 223 breast cancer patients irradiated for BM, Dawood et al. showed that HER2 positive status was an independent

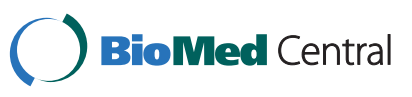


favorable prognostic factor [9]. On the contrary, the triple negative population seems to be associated with worse prognosis $[10,11]$. These results have prompted the development of specific prognostic scores for BM from breast cancer taking into account either tumor phenotypic characteristics [12,13] or not [14]. Given the number of scoring systems that have been devised for clinical use, the aim of our study was to compare the clinical relevance of the major existing prognostic scores in a cohort of breast cancer patients with BM and known HER2 and HR status.

\section{Methods}

\section{Study population}

Medical records of breast cancer patients with BM were retrospectively extracted from the databases of two French cancer centers. Patients were accrued over a 15year period, between 1995 and 2010. Inclusion criteria were as follows: histologically proven breast carcinoma, intradural BM detected by contrast-enhanced cerebral computed tomography or magnetic resonance imaging, and known HR and HER2 status. The tumor was considered HR positive when more than $10 \%$ of cells were labeled in immunohistochemistry (IHC) or when the concentrations of estrogen and progesterone receptors were above $10 \mathrm{ng} / \mathrm{ml}$ and $50 \mathrm{ng} / \mathrm{ml}$ using the radioligand binding method, respectively. The tumor was considered HER2 positive if the primary tumor was scored $3+$ by IHC or if the HER2 gene was amplified by fluorescence in situ hybridization (FISH). If the tumor was scored 2+ by IHC, it was re-analyzed using FISH. Patients with history of other primitive carcinoma or leptomeningeal carcinomatosis were excluded. In addition, an additional brain MRI was performed to all patient presenting with 1 to $3 \mathrm{BM}$ at baseline CT-scan. Clinical data and, when available, biological parameters were extracted in order to score patients using the RPA [1], the GPA [2], the BSBM [3], the P1PS [4], the Breast-GPA [12], the BreastRPA [14] and Le Scodan's score [13], whose constituting parameters are detailed in Table 1. Ethical approval, as well as permission to create, complete and access the comprehensive database used in this study, was provided by the local research ethics committee of the Val d'Aurelle Cancer Institute. Due to the retrospective, non interventional nature of this study, no consent was requested by the local research ethics committee.

\section{Statistical analyses}

Categorical variables were reported by means of contingency tables. For continuous variables, median and range values were computed. To investigate the association between study features, univariate statistical analyses were performed using Pearson's Chi-2 test or Fisher's exact test if applicable for categorical variables. The KruskalWallis test or Student $T$ test were used for continuous
Table 1 Prognostic indexes parameters

A: Clinical parameters used for 5 prognostic indexes (RPA, GPA, BSBM, Breast RPA, and Breast GPA). RPA

\begin{tabular}{|c|c|c|c|c|c|}
\hline Class 1 & \multicolumn{5}{|c|}{$\begin{array}{c}\text { Age }<65 \text { y, KPS } \geq 70 \text {, controlled primary tumor, no } \\
\text { extracranial metastases }\end{array}$} \\
\hline Class 2 & \multicolumn{5}{|c|}{ All patients not in Class I or III } \\
\hline Class 3 & \multicolumn{5}{|c|}{$\mathrm{KPS}<70$} \\
\hline \multicolumn{6}{|c|}{ GPA } \\
\hline & & & 0 & 0,5 & 1 \\
\hline Age & & & $>60$ & $50-59$ & $<50$ \\
\hline KPS & & & $<70$ & $70-80$ & $90-100$ \\
\hline Number of BM & & & $>3$ & $2-3$ & 1 \\
\hline Extracranial meta & stases & & Yes & - & No \\
\hline \multicolumn{6}{|c|}{ BS-BM } \\
\hline & & & & 0 & 1 \\
\hline KPS & & & & $50-70$ & $80-100$ \\
\hline Control of prima & y tumo & & & No & Yes \\
\hline Extracranial meta & stases & & & Yes & No \\
\hline \multicolumn{6}{|c|}{ Breast RPA } \\
\hline Class 1 & \multicolumn{5}{|c|}{$\begin{array}{c}1-2 \text { brain metastases and extracranial disease absent } \\
\text { or controlled and KPS } 100\end{array}$} \\
\hline Class 2 & \multicolumn{5}{|c|}{ All patients not in Class I or III } \\
\hline Class 3 & \multicolumn{5}{|c|}{ Multiple brain metastases and KPS $\leq 60$} \\
\hline \multicolumn{6}{|c|}{ Breast GPA } \\
\hline & 0 & 0,5 & 1 & 1.5 & 2 \\
\hline Age & $\geq 60$ & $<60$ & & & \\
\hline KPS & $\leq 50$ & 60 & $70-80$ & $90-100$ & \\
\hline Genetic subtype & Basal & & Luminal A & HER2 & Luminal B \\
\hline
\end{tabular}

B: Clinico-biological parameters used for the P1PS and Le Scodan's prognostic indexes.

\begin{tabular}{lcc}
\hline & P1PS & \\
\hline & 0 & 1 \\
Sites of metastases & $0-2$ & $>$ ULN \\
Serum LDH & $<$ ULN & $<35$ \\
Albumin, g/L & $\geq 35$ & \\
\hline Class I & He Scodan Score & \\
Class II & All patients not in Class I or III \\
Class III & Tumors not treated with trastuzumab and: \\
& lymphopenia at BM diagnosis or KPS $<70$ \\
& and $\geq 50$ years old at BM diagnosis or KPS $\geq 70$ \\
& and triple negative tumors
\end{tabular}

RPA, Recursive Partitioning Analysis, GPA, Graded Partitioning Analysis, BS-BM, Basic Score for Brain Metastases, BM, Brain Metastases, P1PS, phase 1 prognostic score, KPS, Karnofsky Performance Status, $B M$, Brain Metastases, $L D H$, Lactate Dehydrogenase, ULN, Upper Limit of Normal. 
variables. Overall survival (OS) time was measured from the date of $\mathrm{BM}$ diagnosis to the date of death from any cause. Patients alive without event were censored at the closing date of the study analysis (August 1st, 2011). OS rates and median values were estimated according to the Kaplan-Meier method [15], and presented with their 95\% confidence intervals (95\% CIs). The median length of follow-up was estimated using a reverse Kaplan-Meier method and presented with 95\% CIs.

Pair wise comparisons of subgroups were performed for each score. Survival curves were drawn and the logrank test was performed to assess differences between groups. Harrell's concordance Index ( $C$ index) was used to assess the discriminating ability of the different PIs [16]. To investigate prognostics factors, multivariate analyses were carried out using the Cox's proportional hazards regression model with a stepwise selection procedure $[17,18]$. Hazard ratios (HR) with $95 \%$ CIs are presented to display risk reductions. All $\mathrm{p}$ values reported are two-sided, and the significance level was set at 5\% $(\mathrm{p}<0.05)$. Statistical analysis was performed using the STATA 11 software (Stata Corporation, College Station, TX).

\section{Results}

\section{Patient characteristics}

There were a total of two hundred and fifty patients included in this analysis. Patient characteristics are detailed in Table 2. At the time of BM diagnosis, the median age was 55 years (range 25-85), and 74\% of patients had good performance status $(80-100)$. The brain was the first metastatic site in about one third of patients (34\%), and the only site of metastatic disease in $12 \%$ of patients. Of the 250 patients, $44 \%$ had a primary tumor that over-expressed HER2, while $26 \%$ were diagnosed with a triple negative breast cancer (negative HR and HER2 status). A total of 47 patients (18.8\%) underwent targeted local treatment, namely stereotactic radiotherapy or surgery. Whole brain radiation therapy (WBRT), used as primary treatment but also as adjuvant treatment after localized treatment, was given to 217 patients (86.8\%). Fifteen patients received best supportive care only. After a median follow-up of 4.5 years, the median OS (MOS) was 8.9 months (95\% CI, 6.9-10.3 months). The six-month, one-year and two-year overall survival rates were 61\% (95\% CI, 54-67\%), 40\% (95\% CI, 34-46\%) and $22 \%$ (95\% CI, 17-27\%), respectively.

\section{Prognostic indexes analysis}

Table 3 lists the study population distribution as well as the MOS for each PI. Survival curves are depicted in Figure 1. The results showed that all scores were able to discriminate with statistical significance $(\mathrm{p}<0.001)$ patients for OS according to the prognostic category.
MOS times for the RPA classes I, II and III were 25.6 months (95\% CI 18.4-32.9), 10.4 months (95\% CI 8.912.6), and 2 months (95\% CI 1.4-3.1), respectively. For the GPA classes I, II and III, the MOS were 25.6 months (95\% CI 3.1-5.4), 12.3 months (95\% CI 10.1-15.1), and 24.7 months (95\% CI 12.7-27.1), respectively. In patients stratified in the classes I, II, III and IV using the BS-BM prognostic scores, the MOS were 2.2 months (95\% CI 1.4-3.6), 8.7 months (95\% CI 6.1-12.3), 12.7 months (95\% CI 12.7-27.1), and 21.6 months (95\% CI 12.7-25.6), respectively. With respect to the P1PS, ninety-one patients could not be classified due to missing biological data. The MOS were 16.4 months (95\% CI 11.9-

\section{Table 2 Study population}

\begin{tabular}{|c|c|c|}
\hline Patient characteristics & $\begin{array}{l}\text { Nun } \\
\text { pati }\end{array}$ & $\%$ \\
\hline \multicolumn{3}{|l|}{$\begin{array}{l}\text { Age at breast cancer } \\
\text { diagnosis (years) }\end{array}$} \\
\hline Median, range & \multicolumn{2}{|c|}{$50(23-82)$} \\
\hline \multicolumn{3}{|c|}{ Age at BM diagnosis (years) } \\
\hline Median, range & \multicolumn{2}{|c|}{$55(25-85)$} \\
\hline \multicolumn{3}{|c|}{$\begin{array}{l}\text { Time between initial diagnosis and BM } \\
\text { diagnosis (months) }\end{array}$} \\
\hline Median, range & \multicolumn{2}{|c|}{$39.4(0-319.2)$} \\
\hline \multicolumn{3}{|l|}{ Hormone receptor status } \\
\hline Positive & 119 & 47.6 \\
\hline Negative & 131 & 52.4 \\
\hline \multicolumn{3}{|l|}{ HER-2 status } \\
\hline Positive & 109 & 43.6 \\
\hline Negative & 141 & 56.4 \\
\hline \multicolumn{3}{|c|}{ Karnofsky Performance status } \\
\hline 100 & 66 & 26.4 \\
\hline $80-90$ & 119 & 47.6 \\
\hline $60-70$ & 28 & 11.2 \\
\hline $40-50$ & 32 & 12.8 \\
\hline $10-30$ & 5 & 2 \\
\hline \multicolumn{3}{|l|}{$\begin{array}{l}\text { Extra-cerebral metastases } \\
\text { at BM diagnosis }\end{array}$} \\
\hline Yes & 219 & 87.6 \\
\hline No & 31 & 12.4 \\
\hline \multicolumn{3}{|c|}{ Systemic treatment after BM diagnosis } \\
\hline \multicolumn{3}{|l|}{ Chemotherapy } \\
\hline Yes & 177 & 70.8 \\
\hline No & 73 & 29.2 \\
\hline \multicolumn{3}{|c|}{ Anti-HER2 treatment for HER2+ patients } \\
\hline Yes & 20 & 18.3 \\
\hline No & 89 & 81.7 \\
\hline
\end{tabular}

BM, Brain Metastases, KPS, Karnofsky Performance Status. 
23.3) vs. 5.9 months (95\% CI 3.4-8.4) in remaining patients with P1PS scores of $0 / 1$ vs. $2 / 3$, respectively. Based on the breast GPA scoring system, MOS were found to be 2.3 months for a score of 0-1 (95\% CI 14.1), 5.7 months for a score of 1.5-2.5 (95\% CI 4-8), 10.3 months for a score of 3 (95\% CI 8.4-13.7), and 18.4 months for a score $>3$ (95\% CI 12.4-23.3). The MOS were 21.3 months (95\% CI 9.7-53.9) for class I, 9.8 months (95\% CI 8.4-12.1) for class II, and 2.3 months (95\% CI 1.8-4.3) for class III according to the breast RPA scoring system. Lastly, the MOS for Le Scodan' scores I, II and III were 15.2 (95\% CI 11.5-19.4), 9.7 (95\% CI 7.5-12.4), and 4.2 (95\% CI 3.3-6.1) months, respectively.
Pairwise comparisons of each PI revealed statistically significant differences in survival between prognostic classes except for the breast GPA classes I vs. II ( $p=0.0609)$, the BS-BM scores 1 vs. 2 ( $\mathrm{p}=0.27$ ), and Le Scodan's scores I vs. II $(\mathrm{p}=0.098)$.

\section{Prognostic indexes comparison}

There were no statistically significant differences between all PIs with regard to survival predicting ability (Table 3). Only minor differences were seen using Harrell's concordance index, with values of Harrell's C slightly higher for the BS-BM (0.6803) and RPA (0.6783) scoring systems than for the breast RPA (0.6037), Le Scodan's score (0.6239), and P1PS (0.6251). In

Table 3 Distribution of the study population and median overall survival according to the class of prognostic scores; Harrell's concordance indexes (HCS)

\begin{tabular}{|c|c|c|c|c|c|}
\hline & Number of pts (\%) & $\operatorname{MOS}(95 \% \mathrm{Cl})$ & $p$ & Hazard Ratio $(95 \% \mathrm{CI})$ & $\mathrm{HCS}(95 \% \mathrm{Cl})$ \\
\hline \multicolumn{6}{|l|}{ RPA } \\
\hline 1 & $26(10.4)$ & 25.6 M (18.4-32.9) & & 1 & 0.6783 \\
\hline 2 & $166(66.4)$ & 10.4 M (8.9-12.6) & $<0.001$ & $2.16(1.34-3.50)$ & $(0.65-0.71)$ \\
\hline 3 & $58(23.2)$ & $2 \mathrm{M}(1.4-3.1)$ & & $11.38(6.57-19.70)$ & \\
\hline \multicolumn{6}{|l|}{ GPA } \\
\hline$\geq 3$ & $32(12.8)$ & $24.7 \mathrm{M}(12.7-27.1)$ & & 1 & 0.658 \\
\hline $1.5-2.5$ & $116(46.4)$ & $12.3 \mathrm{M}(10.1-15.1)$ & $<0.001$ & $1.70(1.09-2.65)$ & $(0.62-0.69)$ \\
\hline $0-1$ & $102(40.8)$ & $4.2 \mathrm{M}(3.1-5.4)$ & & $4.15(2.62-6.55)$ & \\
\hline \multicolumn{6}{|l|}{ BS-BM } \\
\hline 3 & $31(12.4)$ & $21.6 \mathrm{M}(12.7-25.8)$ & $<0.001$ & 1 & \\
\hline 2 & $68(27.2)$ & 12.7 M (9.7-18.4) & & $1.30(0.81-2.08)$ & 0.6803 \\
\hline 1 & $96(38.4)$ & $8.7 \mathrm{M}(6.1-12.3)$ & & $2.20(1.41-3.46)$ & $(0.64-0.72)$ \\
\hline 0 & $55(22.0)$ & $2.2 \mathrm{M}(1.4-3.6)$ & & $6.99(4.25-11.47)$ & \\
\hline \multicolumn{6}{|l|}{ P1PS } \\
\hline $0-1$ & $95(38.0)$ & 16.4 M (11.9-23.3) & & 1 & 0.6251 \\
\hline $2-3$ & $64(25.6)$ & $5.9 \mathrm{M}(3.4-8.4)$ & $<0.001$ & $2.89(2.01-4.14)$ & $(0.58-0.66)$ \\
\hline Not available & $91(36.4)$ & & & & \\
\hline \multicolumn{6}{|l|}{ Breast GPA } \\
\hline $3.5-4$ & $53(21.2)$ & 18.4 M (12.4-23.3) & & 1 & 0.6587 \\
\hline 3 & $90(36.0)$ & 10.3 M (8.4-13.7) & $<0.001$ & $1.37(0.95-2.00)$ & $(0.62-0.69)$ \\
\hline $1.5-2.5$ & 76 (30.4) & 5.7 M (4-8) & & $2.09(1.43-3.06)$ & \\
\hline $0-1$ & $31(12.4)$ & $2.3 \mathrm{M}(1-4.1)$ & & $5.75(3.56-9.27)$ & \\
\hline \multicolumn{6}{|l|}{ Breast RPA } \\
\hline 1 & $20(8.0)$ & $21.3 \mathrm{M}(9.7-53.9)$ & & 1 & 0.6037 \\
\hline 2 & $192(76.8)$ & $9.8 \mathrm{M}(8.4-12.1)$ & $<0.001$ & $2.05(1.20-3.50)$ & $(0.57-0.63)$ \\
\hline 3 & $38(15.2)$ & $2.3 \mathrm{M}(1.8-4.3)$ & & $6.84(3.69-12.72)$ & \\
\hline \multicolumn{6}{|c|}{ Le Scodan Score } \\
\hline 1 & 89 (35.6) & 15.2 M (11.5-19.4) & & 1 & 0.6239 \\
\hline 2 & 49 (19.6) & 9.7 M (7.5-12.4) & $<0.001$ & $1.32(0.91-1.92)$ & $(0.58-0.66)$ \\
\hline 3 & $112(44.8)$ & $4.2 \mathrm{M}(3.3-6.1)$ & & $1.92(1.43-2.59)$ & \\
\hline
\end{tabular}

$\mathrm{Cl}$, Confidence Interval. MOS, Median Overall Survival (from brain metastases diagnosis). $M$, months. Pts, patients. 


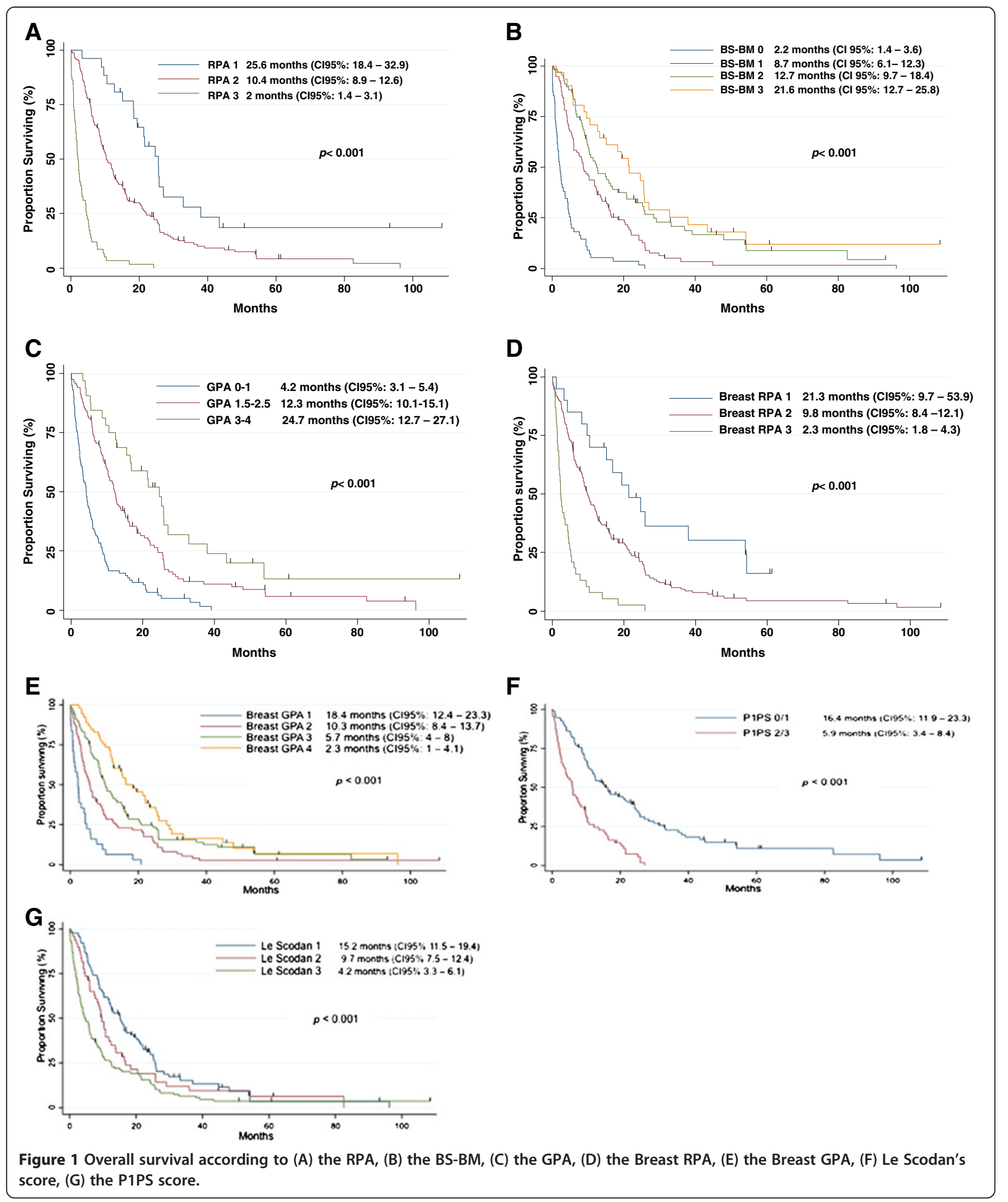




\begin{tabular}{|c|c|c|c|}
\hline a) & HR & $95 \% \mathrm{Cl}$ & $\mathbf{P}$ \\
\hline RPA 2 & 2.76 & $1.64-4.66$ & $<0.001$ \\
\hline RPA 3 & 8.42 & $4.36-16.26$ & $<0.001$ \\
\hline Le Scodan 2 & 1.49 & $1.01-2.19$ & 0.041 \\
\hline Le Scodan 3 & 1.87 & $1.30-2.69$ & 0.001 \\
\hline GPA 3 & 1.75 & $1.22-2.51$ & 0.002 \\
\hline b) & HR & $95 \% \mathrm{Cl}$ & $P$ \\
\hline RPA 2 & 2.42 & $1.36-4.29$ & 0.003 \\
\hline RPA 3 & 13.26 & $6.94-25.30$ & $<0.001$ \\
\hline Le Scodan 3 & 1.57 & $1.03-2.39$ & 0.035 \\
\hline P1PS 2/3 & 2.23 & $1.54-3.24$ & $<0.001$ \\
\hline
\end{tabular}

$R P A$, recursive partitioning analysis, GPA, graded partitioning analysis, $P 1 P S$, phase 1 prognostic score, $H R$, hazard ratio, $\mathrm{Cl}$, confidence interval.

multivariate analysis (excluding P1PS whose data were only available for 159 patients), the RPA, Le Scodan's score, and GPA were found to be the best independent predictors of overall survival. In a second multivariate analysis restricted to the 159 patients with known serum LDH level and proteinemia, the RPA 2 and 3, Le Scodan's score 3 and P1PS 2/3 were associated with worse survival (Table 4).

When evaluating the ability of the different scores to correctly stratify patients with short or long life expectancy, the patients with a MOS longer than 12 months accounted for $85 \%, 75 \%, 71 \%, 70 \%, 68 \%, 58 \%$ and $58 \%$ of the "good prognosis" population defined as RPA 1, GPA $\geq 3$, BS-BM 3, Breast RPA 1, Breast GPA 3.5-4, Le Scodan 1, BS 0-1, respectively. Patients with a MOS shorter than 3 months accounted for $62 \%$, 39\%, 60\%, $58 \%, 61 \%, 37.5 \%$, and $34 \%$ of the "poor prognosis": population defined as RPA 3, GPA 0-1, BS-BM 0, Breast RPA 3, Breast GPA 0-1, Le Scodan 3, BS 2-3, respectively. The misclassification rates in patients living more than 12 months but classified as "poor prognosis" population were $3 \%, 17 \%, 5.5 \%, 8 \%, 6.5 \%, 26 \%$ and $25 \%$, respectively. Conversely, the misclassification rates in patients living less than 3 months but classified as "good prognosis" population were $0 \%, 0 \%, 3 \%, 5 \%, 2 \%, 4.5 \%$ and $7 \%$, respectively.

\section{Discussion}

This comprehensive and simultaneous analysis of 7 prognostic scores was performed on a large, wellcharacterized and homogeneous population of 250 breast cancer patients with BM. This study examined three common scores, namely the RPA, the GPA, and the BS-BM, as well as four new scores incorporating biological or breast-specific parameters: the breast RPA, the breast GPA, Le Scodan's score, and the P1PS. With respect to other scoring systems, the Rotterdam score was not investigated since it uses, as a prognostic variable, the clinical response to steroid therapy prior to panencephalic radiotherapy, which is a subjective information not necessarily collected in clinical observations [5]. In the same way, neither the volume of the largest $\mathrm{BM}$, nor the time between BM diagnosis and the beginning of radiotherapy were available to calculate the SIR [6] and Rades [7] scores, respectively.

Until recently, there have been few studies focusing on $\mathrm{BM}$ prognostic scores in breast cancer. Yet, it has been demonstrated that the reliability and clinical relevance of these scores vary greatly depending on the type of primary tumor. Sperduto et al. found that, in a population of 4,259 patients with 642 breast cancers, the GPA was unfit not only for breast tumor, but also for gastrointestinal, melanoma, and renal cell cancer [8]. Similarly, the widely used RPA index has some limitations in breast disease as it does not consider specific tumor markers, such as the status of HR and HER2. Moreover, the description of extra-cerebral disease is probably not the best suited variable for this pathology, since the prognosis of women with bone metastases or locoregional recurrences differs from that of patients with liver or lung metastases. Recently, efforts have been made to improve accuracy of previous classifications by taking into account breast cancer biomarkers. As such, the GPA score has been replaced by a score specific to breast cancer integrating the status of both HER2 and HR [12]. Likewise, Le Scodan's score, including the breast cancer molecular subtype and treatment parameters, has been proposed from a retrospective analysis of a selected population of patients presenting with advanced disease [12].

Overall, our results indicated that the different scores were able to discriminate the prognosis of patients, which is in keeping with the analysis of Nieder et al. who compared a variety of prognostic classifications from all published trials performed on more than 20 patients [19]. However, the new classifications failed to improve patient selection, with the Breast GPA and Breast RPA scores showing lower Harrell's concordance indexes than the original RPA score. The diversity of populations between studies might explain discrepancies in results and makes generalization difficult. Indeed, the patients analyzed in the Breast GPA pivotal study did not reflect daily clinical practice since $62 \%$ of patients presented 1 to $3 \mathrm{BM}, 35 \%$ had BM without extra-cranial metastases, 37\% were aged less than 50 years, $57 \%$ had tumors overexpressing HER2 receptor, and 68\% of patients received targeted local treatments, which probably explains an impressively good survival (13.8 months). Regarding the results from the Breast RPA pivotal study, in comparison of our study population, the 
irradiation of $98 \%$ of the population represents a selection bias related to the treatment received after BM diagnosis compared to a general clinical practice situation [14]. Contrary to previous indexes, Le Scodan's score had an independent prognostic value in multiparametric analysis, emphasizing the importance of biological subtypes and blood parameters [13]. However, the drawback is that the definition of biological subtype varies depending on the author. Le Scodan et al. distinguished between HER2 positive population treated with trastuzumab and triple negative breast cancer [13], while Sperduto et al. [12] and Niwinska et al. [14] distinguished between luminal A, B, HER2, and basal tumors. In these last two studies, $77 \%$ and $50 \%$ of the HER2+ population were treated using anti-HER2 agents, respectively. It would have been interesting to integrate, as did Le Scodan, the anti-HER2 treatment in the biological subtype since there is increasing evidence that antiHER2 treatments prolong survival of breast cancer patients with BM [9-11,20]. Biological parameters, such as lymphopenia for Le Scodan's score and LDH and proteinemia for the P1PS [4], have been shown to have an independent prognostic value on multiparametric analysis and thus warrant further evaluation. Evaluating subclinical disease activity and the impact on nutritional status may confer additional prognostic information.

One of the strengths of our study is to reflect routine clinical practice population, without selection based on performance status, number of metastases or treatment. This is essential to provide physicians with a clinical tool applicable to the whole patient population at the time of $\mathrm{BM}$ diagnosis. According to our analysis, the RPA score can still be considered as the reference score for several reasons. Firstly, although Harrell's concordance Indexes were quite similar for all PIs, the hazard ratio of the RPA was higher than those of other PIs in multivariate analysis. Our results were consistent with those reported by (i) Le Scodan et al. [21] and Mahmoud-Ahmed et al. [22] who confirmed the prognostic value of the RPA score in the setting of BM from breast cancer (ii) Viani et al. who found a superiority of the RPA score over the BS-BM one [23]. Secondly, one must keep in mind the primary goal of these classifications which is to adapt treatment options to the individual patient prognosis. We need to mitigate the treatment burden for patients with short life expectancy, and conversely to intensify therapeutic interventions for patients for whom an improvement in overall survival is expected. Hence, it is important to know how often the prognostic scores wrongly categorize patients in inappropriate prognosis groups. Nieder et al. studied their ability to correctly classify patients with good prognosis (MOS longer than 6 months from the diagnosis of BM) and patients with poor prognosis (MOS shorter than 2 months from the diagnosis of BM) [24]. In our study, the MOS was 8.9 months and $40 \%$ of the population was alive at 1 year, so we decided to adapt the cut offs used by Nieder to our study population, and we considered boundaries to be a MOS of less than 3 months and a MOS of more than 12 months. In these circumstances, the RPA proved to be more efficient than the other scores to predict median survival since $85 \%$ of patients classified as RPA 1 survived more than 12 months, and $62 \%$ of patients classified as RPA 3 survived less than 3 months. Furthermore, the RPA misclassified a smaller proportion of patients than the other scoring systems as no patients classified RPA 1 survived less than 3 months and only $3 \%$ of patients classified as RPA 3 survived more than 12 months.

A particular weakness of some of the classification systems is the lack of homogeneous distribution of patients between the different prognostic categories. Indeed, a score that would identify a subgroup with excellent prognosis in a very small number of patients, a situation rarely seen in clinical practice, would have limited impact to aid therapeutic decision making in routine practice. This is one of the pitfalls of the GPA scoring since the class 3.5-4 of better prognosis accounts only for $2.8 \%$ of our daily clinical practice population. Finally, an ideal prognostic score should be simple and easily usable in clinical practice. Our analysis at this stage differs from that of Sperduto et al. [2] in so far that we believe that the RPA score is more readily reproducible in practice thanks to a limited number of variables to be collected and fewer prognostic classes.

Nevertheless, due to its retrospective nature, our study suffers some limitations. First, in retrospective analysis, it could be difficult to assess controlled versus uncontrolled distant metastases. As this information is required in Breast RPA prognostic index, the retrospective analysis of this factor could have misclassified some patients. Similarly, a retrospective evaluation of KPS appears less reliable than the evaluation of Performance Status using ECOG classification, and could have led to some degrees of misclassification.

\section{Conclusion}

The new PIs did not perform better than the original scores. Although tumor subtypes, HER2 expression, and blood parameters (LDH, proteinemia, lymphopenia) may have an interesting additional prognostic value, the RPA appears to be the most appropriate and simplest available tool to help clinicians select breast cancer patients with BM.

\section{Abbreviations}

BM: Brain metastases; BS-BM: Basic score for brain metastases; GPA: Graded prognostic assessment; HER2: Human epidermal growth factor receptor-2; HR: Hormonal receptor; IHC: Immunohistochemistry; KPS: Karnofsky performance status; LDH: Lactate dehydrogenase; MOS: Median overall 
survival; OS: Overall survival; P1PS: Phase 1 prognostic score; PI: Prognostic indexes; RPA: Recursive partitioning analysis; SIR: Score index for radiosurgery; ULN: Upper limit of normal.

\section{Competing interests}

The authors declare that they have no conflict of interest.

\section{Authors' contributions}

Conception and design: ALB, WJ, D. Provision of study material or patients: ALB, WJ, DA, J-MF, GR. Collection and assembly of data: ALB, WJ, ST. Data analysis and interpretation: ALB, WJ, DA, ST. Manuscript writing: ALB, WJ, DA, ST. Final approval of the manuscript: ALB, WJ, DA, J-MF, GR, ST.

\section{Acknowledgment}

The authors are grateful to Mrs Vanessa Guillaumon for her technical assistance in the manuscript writing process.

\section{Author details}

${ }^{1}$ Department of Radiation Oncology, Val d'Aurelle Cancer Institute, 208 rue des apothicaires, Montpellier 34298, France. ${ }^{2}$ Department of Biostatistics, Val d'Aurelle Cancer Institute, 208 rue des apothicaires, Montpellier 34298, France. ${ }^{3}$ Department of Medical Oncology, Val d'Aurelle Cancer Institute, 208 rue des apothicaires, Montpellier 34298, France. ${ }^{4}$ Department of Medical Oncology, Antoine Lacassagne Cancer Institute, 33, avenue de Valombrose, Nice Cedex 02 06189, France.

Received: 31 July 2012 Accepted: 28 January 2013

Published: 8 February 2013

\section{References}

1. Gaspar L, Scott C, Rotman M, Asbell S, Phillips T, Wasserman T, McKenna WG, Byhardt R: Recursive partitioning analysis (RPA) of prognostic factors in three Radiation Therapy Oncology Group (RTOG) brain metastases trials. Int J Radiat Oncol Biol Phys 1997, 37(4):745-751.

2. Sperduto PW, Berkey B, Gaspar LE, Mehta M, Curran W: A new prognostic index and comparison to three other indices for patients with brain metastases: an analysis of 1,960 patients in the RTOG database. Int J Radiat Oncol Biol Phys 2008, 70(2):510-514.

3. Lorenzoni J, Devriendt D, Massager N, David P, Ruiz S, Vanderlinden B, Van Houtte P, Brotchi J, Levivier M: Radiosurgery for treatment of brain metastases: estimation of patient eligibility using three stratification systems. Int J Radiat Oncol Biol Phys 2004, 60(1):218-224.

4. Nieder C, Dalhaug A: A new prognostic score derived from phase I study participants with advanced solid tumours is also valid in patients with brain metastasis. Anticancer Res 2010, 30(3):977-979.

5. Lagerwaard FJ, Levendag PC, Nowak PJ, Eijkenboom WM, Hanssens PE, Schmitz PI: Identification of prognostic factors in patients with brain metastases: a review of 1292 patients. Int J Radiat Oncol Biol Phys 1999, 43(4):795-803.

6. Weltman E, Salvajoli JV, Brandt RA, De Morais HR, Prisco FE, Cruz JC, De Oliveira Borges SR, Wajsbrot DB: Radiosurgery for brain metastases: a score index for predicting prognosis. Int J Radiat Oncol Biol Phys 2000, 46(5):1155-1161.

7. Rades D, Dziggel L, Haatanen T, Veninga T, Lohynska R, Dunst J, Schild SE: Scoring Systems to Estimate Intracerebral Control and Survival Rates of Patients Irradiated for Brain Metastases. Int J Radiat Oncol Biol Phys 2011, 80(4):1122-1127.

8. Sperduto PW, Chao ST, Sneed PK, Luo X, Suh J, Roberge D, Bhatt A, Jensen AW, Brown PD, Shih $H$, et al: Diagnosis-specific prognostic factors, indexes, and treatment outcomes for patients with newly diagnosed brain metastases: a multi-institutional analysis of 4,259 patients. Int J Radiat Oncol Biol Phys 2010, 77(3):655-661.

9. Dawood S, Broglio K, Esteva FJ, Ibrahim NK, Kau SW, Islam R, Aldape KD, Yu TK, Hortobagyi GN, Gonzalez-Angulo AM: Defining prognosis for women with breast cancer and CNS metastases by HER2 status. Ann Oncol 2008, 19(7):1242-1248

10. Nam BH, Kim SY, Han HS, Kwon Y, Lee KS, Kim TH, Ro J: Breast cancer subtypes and survival in patients with brain metastases. Breast Cancer Res 2008, 10(1):R20.

11. Niwinska A, Murawska M, Pogoda K: Breast cancer brain metastases: differences in survival depending on biological subtype, RPA RTOG prognostic class and systemic treatment after whole-brain radiotherapy (WBRT). Ann Oncol 2010, 21(5):942-948.

12. Sperduto PW, Kased N, Roberge D, XU Z, Shanley R, Luo X, Sneed PK, Chao ST, Weil RJ, Suh J, et al: Effect of Tumor Subtype on Survival and the Graded Prognostic Assessment for Patients With Breast Cancer and Brain Metastases. Int J Radiat Oncol Biol Phys 2012, 82(5):2111-2117.

13. Le Scodan R, Massard C, Jouanneau L, Coussy F, Gutierrez M, Kirova Y, Lerebours F, Labib A, Mouret-Fourme E: Brain metastases from breast cancer: proposition of new prognostic score including molecular subtypes and treatment. J Neuroonco/ 2012, 106(1):169-176.

14. Niwinska A, Murawska M: New Breast Cancer Recursive Partitioning Analysis Prognostic Index in Patients with Newly Diagnosed Brain Metastases. Int J Radiat Oncol Biol Phys 2012, 82(5):2065-2071.

15. Kaplan EL, Meier P: Nonparametric estimation from incomplete observations. J Am Stat Assoc 1958, 53:457-481.

16. Koziol JA, Jia Z: The concordance index $C$ and the Mann-Whitney parameter $\operatorname{Pr}(X>Y)$ with randomly censored data. Biom J 2009, 51(3):467-474.

17. Cox DR: The analysis of binary data. London: Methuen and Company; 1970.

18. Cox DR: Regression models and life tables. J R Stat Soc B 1972, 34:187-220.

19. Nieder C, Mehta MP: Prognostic indices for brain metastases-usefulness and challenges. Radiat Oncol 2009, 4:10.

20. Bartsch R, Rottenfusser A, Wenzel C, Dieckmann K, Pluschnig U, Altorjai G, Rudas M, Mader RM, Poetter R, Zielinski CC, et al: Trastuzumab prolongs overall survival in patients with brain metastases from Her2 positive breast cancer. J Neurooncol 2007, 85(3):311-317.

21. Le Scodan R, Massard C, Mouret-Fourme E, Guinebretierre JM, Cohen-Solal C, De Lalande B, Moisson P, Breton-Callu C, Gardner M, Goupil A, et al: Brain metastases from breast carcinoma: validation of the radiation therapy oncology group recursive partitioning analysis classification and proposition of a new prognostic score. Int J Radiat Oncol Biol Phys 2007, 69(3):839-845.

22. Mahmoud-Ahmed AS, Suh JH, Lee SY, Crownover RL, Barnett GH: Results of whole brain radiotherapy in patients with brain metastases from breast cancer: a retrospective study. Int I Radiat Oncol Biol Phys 2002, 54(3):810-817.

23. Viani GA, Castilho MS, Salvajoli JV, Pellizzon AC, Novaes PE, Guimaraes FS, Conte MA, Fogaroli RC: Whole brain radiotherapy for brain metastases from breast cancer: estimation of survival using two stratification systems. BMC Cancer 2007, 7:53.

24. Nieder C, Molls M: Validation of graded prognostic assessment index for patients with brain metastases: in regard to Sperduto et Al (Int J Radiat Oncol Biol Phys 2008;70:510-514). Int J Radiat Oncol Biol Phys 2008, 72(5):1619. author reply 1619.

doi:10.1186/1471-2407-13-70

Cite this article as: Braccini et al:: Comparative performances of prognostic indexes for breast cancer patients presenting with brain metastases. BMC Cancer 2013 13:70.

\section{Submit your next manuscript to BioMed Central and take full advantage of:}

- Convenient online submission

- Thorough peer review

- No space constraints or color figure charges

- Immediate publication on acceptance

- Inclusion in PubMed, CAS, Scopus and Google Scholar

- Research which is freely available for redistribution

Submit your manuscript at www.biomedcentral.com/submit

C) Biomed Central 